\title{
Recent Advances in Fast Multipole Methods to Simulate Ever Larger and More Complex Structures
}

\author{
Femke Olyslager $^{1}$, Kristof Cools ${ }^{2}$, Ignace Bogaert ${ }^{3}$, Jan Fostier ${ }^{4}$, Joris Peeters ${ }^{5}$, Francesco P. Andriulli ${ }^{6}$, \\ Eric Michielssen ${ }^{7}$ \\ ${ }^{1}$ Ghent University, Dept. of Information Technology, Ghent, Belgium, femke.olyslager@intec.ugent.be \\ ${ }^{2}$ Ghent University, Dept. of Information Technology, Ghent, Belgium, kristof.cools@intec.ugent.be \\ ${ }^{3}$ Ghent University, Dept. of Information Technology, Ghent, Belgium, ignace.bogaert@intec.ugent.be \\ ${ }^{4}$ Ghent University, Dept. of Information Technology, Ghent, Belgium, jan.fostier@intec.ugent.be \\ ${ }^{5}$ Ghent University, Dept. of Information Technology, Ghent, Belgium, joris.peeters@ intec.ugent.be \\ ${ }^{6}$ University of Michigan, Dept. of Elec. Eng. and Comp. Science, Ann Arbor, Michigan, fandri@umich.edu \\ ${ }^{7}$ University of Michigan, Dept. of Elec. Eng. and Comp. Science, Ann Arbor, Michigan, emichiel@umich.edu
}

\begin{abstract}
In this paper we wish to focus on some recent advances in the Multilevel Fast Multipole Algorithm (MLFMA). Three different topics will be discussed briefly: a seamless extension of the MLFMA to low frequencies, an asynchronous parallelization of the MLFMA suitable for grid computing environments and a new Calderón based preconditioner for the Electric Field Integral Equation (EFIE). This will be illustrated by three scattering examples in frequency and time domain.
\end{abstract}

\section{INTRODUCTION}

Since the introduction of the MLFMA the use of integral equations has seen a new impetus [1]. The MLFMA drastically reduces the computational and memory complexity of Method of Moments (MoM) discretizations for boundary integral equations from $O\left(N^{2}\right)$ to $O(N \log N)$ with $N$ the number of unknowns in the discretization. At the same time the numerical error is fully controlled in MLFMA. The MLFMA has been used to simulate problems involving several millions of unknowns [2], [3] and in two dimensions problems of tens of thousands wavelengths in size [4].

In [5] we have concentrated on the application of MLFMA for EMC shielding problems. Here, we will focus on a number of recent advances in the MLFMA. First we will investigate a stable and seamless extension [6] of the classical MLFMA to low frequencies resulting in the first truly broadband MLFMA that does not rely on directional translation operators. As a second extension we briefly discuss a new parallelization scheme [3] for the MLFMA. Contrary to existing schemes the new scheme is asynchronous and very well suited to handle multiple object scattering. Finally we discuss a new preconditioning method based on the Calderón identities for the Electric Field Integral Equation (EFIE) [7], [8]. This method was introduced for time domain integral equations but is also suited for low frequency EFIE frequency domain problems.

We will illustrate these advances by a few numerical examples in two and three dimensions and in frequency and time domain. For more examples we refer to the presentation during the conference and to the cited literature. Most of the code that was implemented to test these new advances is available as open source software under GPL licence [9].

\section{THE NSPWMLFMA}

The Nondirective Stable Plane Wave Multilevel Fast Multipole Algorithm (NSPWMLFMA [6]) is a novel method for calculating the low frequency (LF) interactions that cannot be handled by the classical MLFMA. An interaction is called LF if the source and observer are closer to each other than approximately one wavelength. To expand fields this close to the source into plane waves in a stable manner, evanescent plane waves are necessary. The MLFMA uses only propagating plane waves and therefore succumbs to a severe numerical instability when dealing with LF interactions. Alternative algorithms can be used to deal with the LF interactions. The most widely used ones are based on multipoles [10] or the spectral decomposition of the Green function [11], [12]. However, the former has the disadvantage that the translations are not diagonal and the latter needs six radiation patterns to cover all possible translation directions. As a consequence these methods are computationally suboptimal.

The NSPWMLFMA aims to combine the strengths of the multipole and spectral methods and do away with the weaknesses. It does so by using a novel LF-stable addition theorem for translations in the $z$-direction. This addition theorem employs evanescent plane waves, hence its stability. However, in contrast to the spectral methods, it is still based on the same fundamental formula as the one underlying the MLFMA. Therefore it shares the MLFMA's convergence characteristics and requires only one radiation pattern. There is one problem with this addition theorem, though. It is numerically stable only for translations in $z$-direction. Therefore it is necessary to rotate the coordinate system such that the vector connecting the centers of the interacting source and observer boxes is parallel to the $z$-axis. This causes the radiation patterns to be rotated too, and this in turn causes the discretization points of the radiation patterns to be different for every translation direction. This problem is solved by using the QR-algorithm to select a special set of sample points (wave vectors in which to evaluate the radiation pattern). These sample points are chosen such that the samples contain sufficient information to fully describe the radiation pattern, i.e. the samples can be solved for the multipole coefficients of the radiation pattern. The QR selection procedure of the sample points makes sure that 
this operation is well conditioned. The knowledge of the multipole coefficients then allows the calculation of the radiation pattern in all other possible sample points. This enables the construction of an interpolation matrix that converts the selected samples of the radiation pattern into the required sample points for a certain translation. This matrix is then absorbed into the translation operator for this direction. In this way, numerically stable translation operators are found for all translation directions. The set of selected sample points defines the single radiation pattern.

The LF equivalents of inter- and anterpolations are done by means of dense matrices. This is a disadvantage of the NSPWMLFMA since the size of these matrices grows quadratically with the electric size of the boxes. Therefore, the NSPWMLFMA is an LF technique. It can, however, be easily coupled seamlessly with the MLFMA to obtain a broadband method. Also, the DC limit of the algorithm exists, which clearly shows its LF stability. It is noteworthy that recently a new version of the NSPWMLFMA was created that uses translation operators that are known in closed form [13].

\section{ASYNCHRONOUS PARALlELIZATION}

The current trend in computer architecture is to incorporate several "cores" into a single processing unit (CPU) [14]. These cores run independently and can hence be seen as a parallel system. With the ever dropping hardware prices, several computers can be connected using a cheap but fast interconnection network such as Gigabit Ethernet. It is clear that, in order to take advantage of this increase of computational power, the traditional serial algorithms need to be modified to run in such a distributed environment.

Previous efforts towards distributed parallel MLFMA were largely focused on scattering from very large $3 \mathrm{D}$ PEC objects. Using advanced load balancing schemes and fast interconnection networks, problems with a very large number of unknowns have been demonstrated [2]. These implementations are essentially synchronous and are characterized by alternating phases of calculation and communication. Attempts for such an approach on Gigabit Ethernet networks led to a poor efficiency.

Recently, we proposed an asynchronous approach to the parallelization of the MLFMA [3]. The term "asynchronous" denotes that different processes can execute different types of operations at a given point in time. While some nodes are communicating, others could be calculating, leading to a better spreading of communication through time. This alleviates the need for expensive interconnection networks and avoids communication in bursts. Futhermore, this approach allows for an efficient parallelization of simulations that comprise multiple dielectric objects. The asynchronous MLFMA has been applied to both two dimensional (TE/TM) and three dimensional problems. The source code of these solvers can be obtained free of charge [9].

\section{PReconditioning}

The linear systems of equations resulting from the discretization of integral equations are often ill-conditioned. This is especially cumbersome for the application of MLFMA since it will drastically increase the number of iterations in the iterative solution process. Hence, reducing the condition number by using preconditioning strategies is of paramount importance when wishing to reduce the computational load of MLFMA. Ill-conditioned systems can stem from different origins. On the one hand the physical geometry of the scatterer itself can induce high condition numbers. This will be the case when strong interference effects are encountered such as in e.g. photonic crystals. Dedicated preconditioners can be developed to reduce the condition number [15]. On the other hand the used integral equation itself can be the cause of large condition numbers. This is e.g. the case with the EFIE when increasing the density of the mesh. This is especially worrysome when simulations over wide frequency bands are necessary with a constant mesh or when dealing with non-uniform meshes.

The condition number of EFIE can be improved by constructing a so-called Calderón preconditioner (see e.g. [16]). If $T[\mathbf{j}]$ represents the integral operator in the EFIE acting on the unknown current density $\mathbf{j}$ on the surface of the scatterer then the integral equation can be written compactly as $0=\mathbf{u}_{n} \times \mathbf{e}^{i}(\mathbf{r})+T[\mathbf{j}](\mathbf{r})$ for a PEC with $\mathbf{e}^{i}$ the incident electric field and $\mathbf{u}_{n}$ the unit vector normal to the scatterer's surface. Calderón preconditioning amounts to operating $T$ on this EFIE resulting in the Calderón preconditioned EFIE (CP-EFIE) $0=T\left[\mathbf{u}_{n} \times \mathbf{e}^{i}\right](\mathbf{r})+$ $T^{2}[\mathbf{j}](\mathbf{r})$. It can be shown using the Calderón identities that $T^{2}$ has a bounded singular value spectrum when the frequency or the mesh density decreases.

Discretizing the $T^{2}$ operator by a direct Galerkin method is impractical. Therefore a discretization is used that considers a product of two discretized operators $T$. In the classical MoM for the EFIE the domain of $T$ is discretized using RWG basis functions and the rang using curl-conforming RWG basis functions (i.e. $\mathbf{u}_{n} \times R W G$ basis functions). This cannot be used twice for $T^{2}$ since a singular Gram matrix is encountered when projecting the range of the first operator on the domain of the second operator. Recently, [17], [7], [8], it was shown that this can be resolved elegantly by discretizing the domain of the second $T$ operator in so-called Buffa-Christiansen basis functions (BC basis functions) [17] and the range in $\mathbf{u}_{n} \times B C$ basis functions.

\section{2D FREQUENCY DOMAIN EXAMPLE}

As a two dimensional example, we consider an indoor propagation example. The geometry consists of a wooden $\left(\epsilon_{r}=3.5\right)$ office cubicle with metal (PEC) supports in which metal (PEC) cupboards have been placed. The size of each cubicle is $2 m \times 2 m$. A TM line current operating at $6 \mathrm{GHz}$ is used to excite the structure. The total number of unknowns using a $\lambda / 10$ discretization is 48045 . Using four AMD Opteron 270 cores, the iterative solution took 258 s and 726 iterations to converge to a tolerance of $10^{-3}$. A $1 \lambda \times 1 \lambda$ block-Jacobi preconditioner was used. 


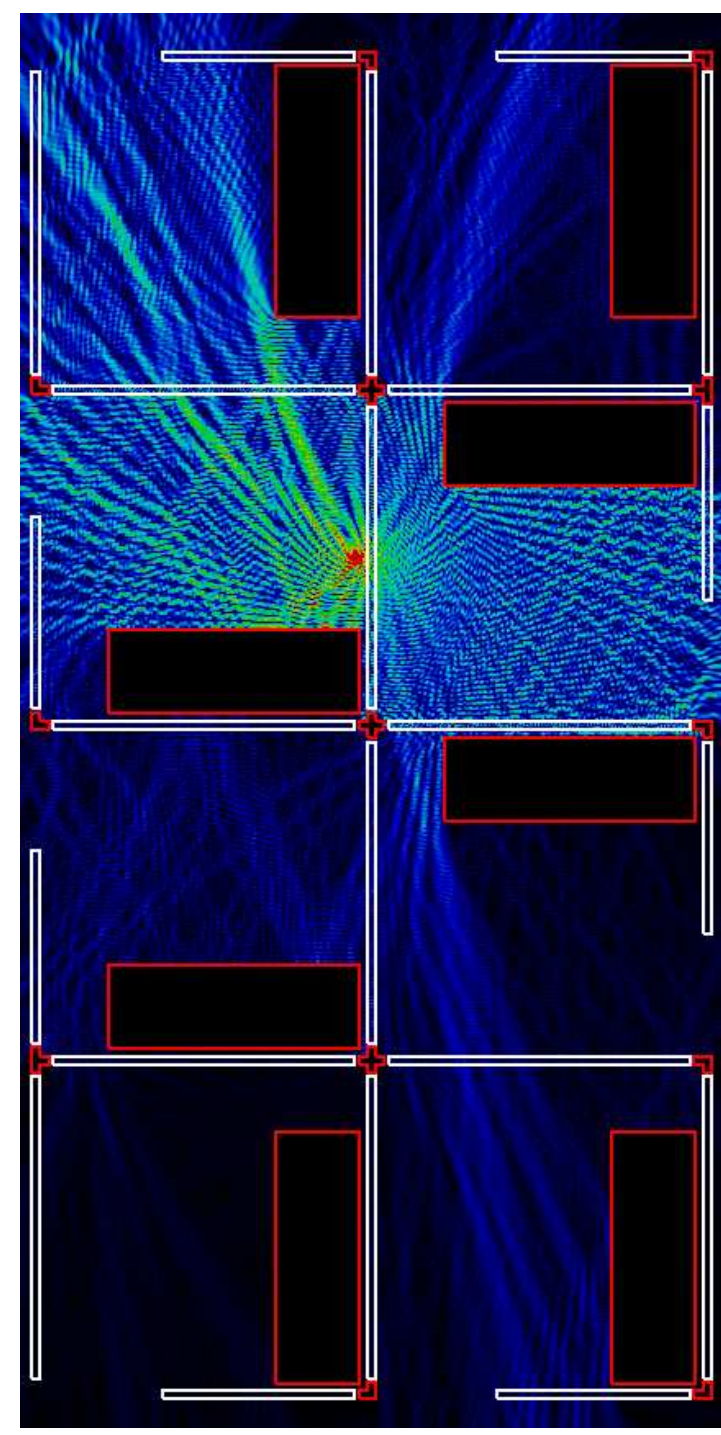

Fig. 1. Electric field density in an office cubicle.

Fig. 1 shows the electrical field density inside the cubicle. Although the number of unknowns is rather modest in this example we have used the same technique to simulate problems with 15000000 of unknowns [3]. We have also used it for passive optical set-ups [18].

\section{3D FREQUENCY DOMAIN EXAMPLE}

As a three dimensional example, we consider the broadband scattering at PEC objects, in casu "Thunderbird 2". First we illuminate a formation of three Thunderbirds with a frontal linearly polarized wave at a frequency such that the length of a Thunderbird corresponds to 15 wavelengths. The electric component of the incident wave is orthogonal to the wings, i.e. vertical. This problem is simulated with the CFIE to avoid spurious reflections and discretized using 1025559 unknowns. It was simulated in about 100 iterations on 20 AMD Opteron 270 cores to a tolerance of $10^{-3}$. The problem required 20 times

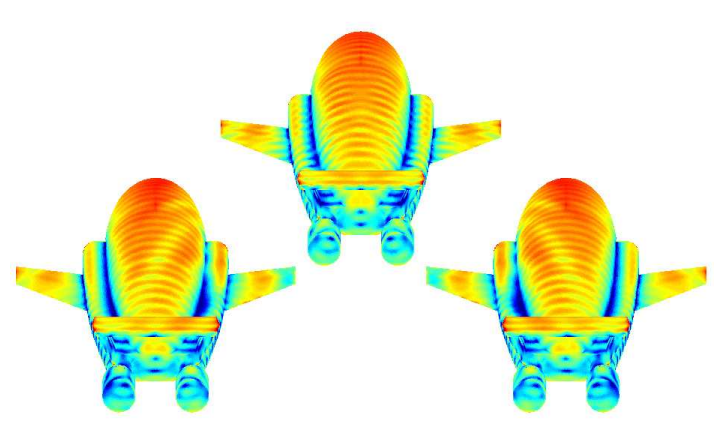

Fig. 2. HF current densities on the surface of a formation of three Thunderbirds.

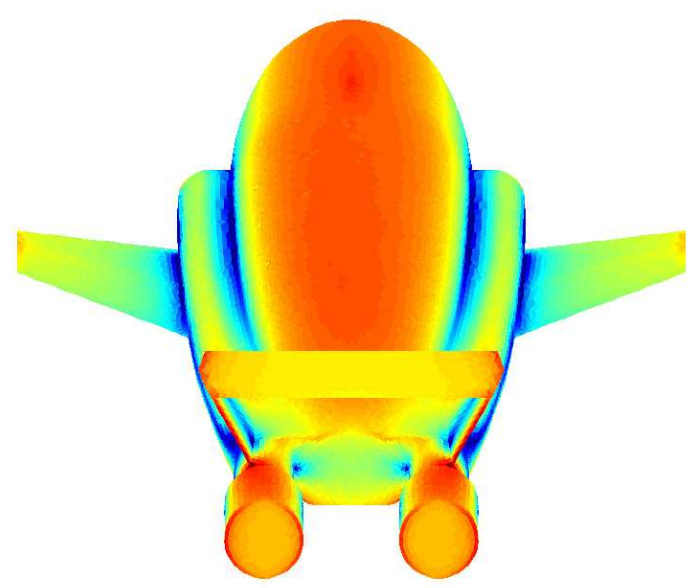

Fig. 3. LF current densities on the surface of one Thunderbird.

1.2GByte of memory and each iteration lasted 28s. Fig. 2 shows the current distribution on the surface of the formation.

To demonstrate the efficacy of the NSPWMLFMA one single Thunderbird was illuminated by the same plane wave but now at a frequency where the length of the Thunderbird is 0.014 wavelengths. For stability reasons a MFIE was used that was discretized with 101466 unknowns. Using 12 processors each iteration took $20 \mathrm{~s}$ and convergence to a tolerance of $10^{-3}$ was reached after 21 iterations. Fig. 3 shows the current distribution.

\section{3D TIME DOMAIN EXAMPLE}

As a last example we consider a 3D scattering problem in time domain. A plane wave time pulse is incident on the PEC structure of Fig. 4. This problem is analyzed with a time domain EFIE and illustrates the capabilities of a Calderón preconditioner and the use of $\mathrm{BC}$ basis functions. Without preconditioner this problem requires 300 iterations per time step and with preconditioner this reduces to 15 iterations. A typical time response for the current density on the structure is shown in Fig. 5. We see that the result of the non-preconditioned and preconditioned solution coincide. The frequency response is shown in Fig. 6. Note the two adjacent resonances 


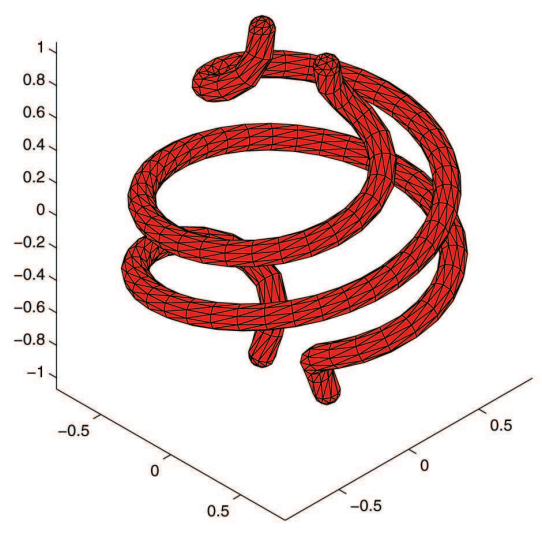

Fig. 4. Geometry and mesh of two intertwined spiral conductors.

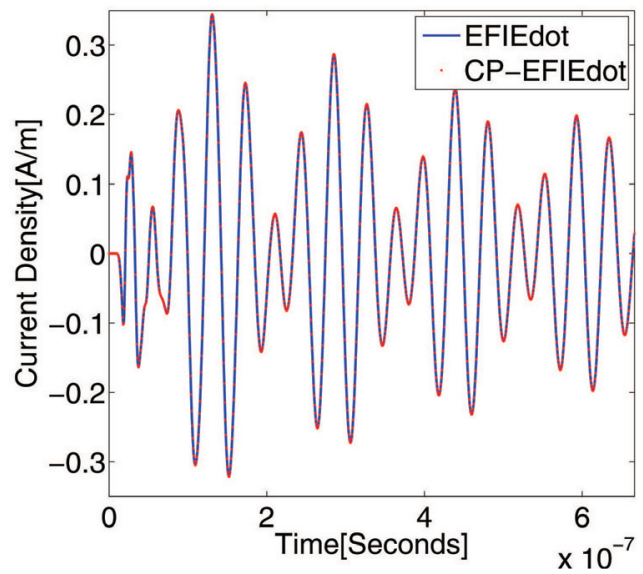

Fig. 5. Time response of the current density due to a pulse incident on the structure of Fig. 4.

that cause the beating phenomenon in Fig. 5. For more information and other examples we refer to [7] and [8].

\section{CONCLUSIONS}

Three different recent advances in the development of the MLFMA have been illustrated. We are convinced that further challenging advances in the MLFMA will ultimately result in a broadband parallel algorithm that allows for the simulation of three-dimensional problems of thousands of wavelengths in size comprising several billions of unknowns on affordable computer networks.

\section{REFERENCES}

[1] W.C. Chew, J.M. Jin, E. Michielssen, and J. Song, Fast and Efficient Algorithms in Computational Electromagnetics, Boston: Artech House, 2001.

[2] L. Gürel and Ö. Ergül, Fast and accurate solutions of extremely large integral-equation problems discretised with tens of millions of unknowns, Electronics Letters, vol. 43, no. 9, pp. 499-500, Apr. 2007.

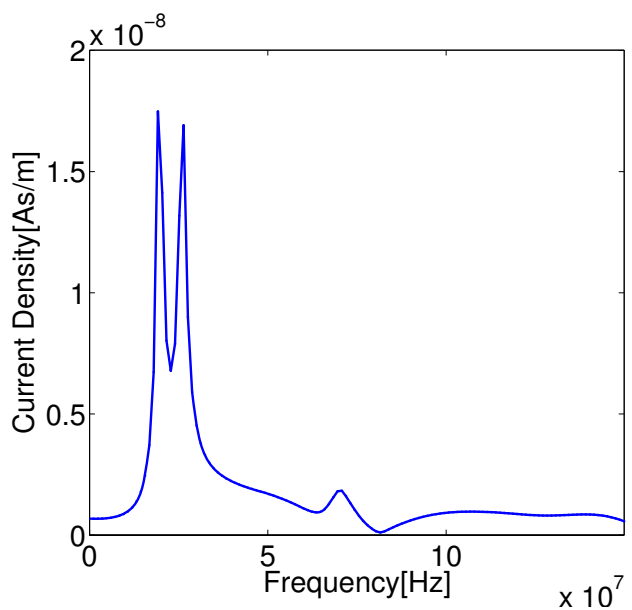

Fig. 6. Frequency response of Fig. 4.

[3] J. Fostier and F. Olyslager, "An asynchronous parallel MLFMA for scattering at multiple dielectric objects," Accepted for IEEE Trans. on Antennas and Propagat.

[4] J. Fostier and F. Olyslager, "A Fast 2.5D Parallel Multilevel Fast Multipole Algorithm Solver," Submitted to Radio Science.

[5] J. Peeters, I. Bogaert, J. Fostier and F. Olyslager, "Accurate Wideband Evaluation of the Shielding Effectiveness of Complex Enclosures Using an Asynchronous Parallel NSPWMLFMA," Accepted for the 19th International Zurich Symposium on Electromagnetic Compatibility, Singapore, May. 2008.

[6] I. Bogaert, J. Peeters, and F. Olyslager, "A nondirective plane wave MLFMA stable at low frequencies," Submitted for IEEE Trans. on Antennas and Propagat. .

[7] K. Cools, F.P. Andriulli, F. Olyslager and E. Michielssen, "Timedomain Calderón identities and their application to the integral equation analysis of scattering by PEC objects part I: preconditioning," Submitted to IEEE Transactions on Antennas and Propagation.

[8] F.P. Andriulli, K. Cools, F. Olyslager and E. Michielssen, "Timedomain Calderón identities and their application to the integral equation analysis of scattering by PEC objects part II: stability," Submitted to IEEE Transactions on Antennas and Propagation.

[9] J. Fostier, J. Peeters, and F. Olyslager, "Open FMM", [Online]. Available: http://openfmm.intec.ugent.be.

[10] L.J. Jiang and W.C. Chew, "A mixed-form fast multipole algorithm," IEEE Transactions on Antennas and Propagation, Vol. 53, no. 12, pp. 4145-4156, 2005.

[11] E. Darve and P. Havé, "A fast multipole method for Maxwell equations stable at all frequencies," Phil. Trans. R. Soc. Lond. A, Vol. 362, pp. 603-628, 2004.

[12] H. Cheng, W.Y. Crutchfield, Z. Gimbutas, L.F. Greengard, J.F Ethridge, J. Huang, V. Rokhlin, N. Yarvin and J. Zhao, "A wideband fast multipole method for the Helmholtz equation in three dimensions", Journal of Computational Physics, no. 216, pp. 300-325, 2006 .

[13] I. Bogaert and F. Olyslager, "A low frequency stable plane wave addition theorem," Submitted to Journal of Computational Physics .

[14] P. F. Gorder, Multicore processors for science and engineering, IEEE Comput. Sci. Eng., vol. 9, no. 2, pp. 37, 2007.

[15] D. Pissoort, E. Michielssen, D. Vande Ginste and F. Olyslager, "A Rank-revealing Preconditioner for the Fast Integral-equation-based Characterization of Electromagnetic Crystal Devices," Microwave and Optical Technology Letters, Vol. 48, no. 4, pp. 783-798, Apr. 2006.

[16] R. Adams and N. Champagne, "A numerical implementation of a modified form of the electric field integral equation," IEEE Trans. Antennas and Propagation, vol. 52, no. 9, pp. 2262-2266, 2004.

[17] A. Buffa and S.H. Christiansen, "A dual finite lement complex on the barycentric refinement," Tech. Report PV-18 IMATI_CNR, 2005.

[18] J. Fostier and F. Olyslager, "A GRID computer implementation of the multilevel fast multipole algorithm for full-wave analysis of optical devices," IEICE Transactions on Communication Vol. E90-B, no. 9, pp. 2430-2438, 2007. 